\title{
One-shot measurement of thermal and kinematic fields from Infra-Red Image Correlation (IRIC)
}

\author{
A. Maynadier ${ }^{1, a}$, M. Poncelet ${ }^{2}$, K. Lavernhe-Taillard ${ }^{1}$, and S. Roux ${ }^{1}$ \\ 1 LMT Cachan, ENS Cachan/CNRS UMR 8535/Univ. Paris 6/PRES UniverSud Paris, 61 Avenue du \\ Président Wilson, 94235 Cachan Cedex, France \\ 2 CEA Saclay, DEN-DANS/DMN/SRMA/LC2M, 91191 Gif sur Yvette Cedex, France
}

\begin{abstract}
Many materials are concerned by strain localization, for instance PLC phenomena, Lüders' bands or Shape Memory Alloys (SMA). The experimental identification of such material behaviors requires the use of full field kinematic measurements, such as provided by Digital Image Correlation (DIC), as well as Infra-Red (IR) thermography to evaluate the associate thermal dissipation. Jointly, these field measurements allow for a full thermo-mechanical characterization of material behavior. However, the space and time association of both fields remains a major difficulty (antagonist surface texture requirements, imaging devices having different pixel number and acquisition rate...). In this paper, we introduce a much simpler experimental approach, which consists in a novel extended DIC technique applied to a single set of IR images. It gives access to both displacement and temperature fields decomposed over the same discretization. This technique, applied to tensile tests on a NiTi SMA, reveals both strain localization due to the phase transformation and associated thermal dissipation.
\end{abstract}

\section{Introduction}

Identification of the constitutive laws of solids prone to localization (Portevin-Le Chatelier phenomena, Lüders' bands, Shape Memory Alloys, metallic glasses, semi-brittle materials, ...) represents a formidable challenge, in particular on the experimental side. Global strain measurements - because of their averaging nature - and local ones — because of their limited sampling capabilities — betray the inherent complexity of the actual strain field, and hence cannot be trusted. Full-field measurements have the unique advantage of offering a global and yet spatially resolved picture of the strain field, (down to a specific resolution). In the following, the considered full field kinematic analysis will be restricted to Digital Image Correlation (DIC).

Moreover, in most cases, strain localization gives rise to a significant thermal dissipation, which calls for thermal or calorimetric measurements to have a more complete characterization of the phenomena at play. Thermography from Infra-Red (IR) camera again provides a global and spatially resolved field measurement from which dissipation can be inferred.

Jointly, these two field measurements open an avenue to the identification of a full thermomechanical description of the material behavior. However, one major difficulty is that these two techniques require different (or even contradictory) surface texture properties: high emissivity homogenous coating for a constitutive measure of temperature using IR camera and a sufficiently contrasted speckle-pattern for DIC. Moreover, these two techniques require also different imaging devices having each their own specific optics, pixel number and acquisition rate. Solutions have been proposed by different groups exploiting clever experimental set-ups, like measurement on both faces of flat sample [1], with proper space and time association of both thermal and mechanical fields. Other groups [2]

a e-mail: anne.maynadier@lmt.ens-cachan.fr

This is an Open Access article distributed under the terms of the Creative Commons Attribution-Noncommercial License 3.0, which permits unrestricted use, distribution, and reproduction in any noncommercial medium, provided the original work is properly cited. 
worked on specimen preparation to perform temperature and displacement measurement on the same face ; they however still use two different imaging devices and consequently still need space and time association of the obtained fields that may constitute a spurious loss of information. Another solution was proposed by [3] by using two visible and near IR cameras and a projected speckle-pattern. The main limitation is that only the out-of-plane displacement and thermal field can be obtained at the same place.

The present paper aims at introducing a much simpler experimental approach which consists in an extended DIC technique applied to a single set of images from an IR camera. In order to be able to follow the displacements field an original speckle-pattern with sufficient contrast in the IR images was developed. This peculiar sample preparation and experimental set-up is described in the first part of this paper. Then, to solve the thermal problem caused by the non homogenous emissivity, a temperature calibration using a cooling test is presented. After this preliminary step, we introduce the equations of the IR image correlation, providing both displacements and temperature fields decomposed on the same Q4 elements basis. This new approach is finally applied to tensile tests on a Nickel-Titanium Shape Memory Alloy (SMA) well known to present martensitic transformation bands with severe inhomogeneous strains and thermal dissipation [4], [5].

\section{Contrasted Infra-Red images : specimen preparation and experimental setup}

In our case, the specimen consists in a thin bone sample with sufficient surface with respect to imaging device. As well as the speckle-pattern contrast is of crucial interest to determine the displacements in DIC, this technique main challenge is firstly to obtain a sufficiently contrasted speckle-pattern for the IR imaging device. Since the contrast is linked to emissivity, we decided to cover our sample surface with strong differences of emissivity.

The retained principle is the following: sample surface underwent an electrochemical polishing, inducing a very poor emissivity. Then, the gauge zone surface is covered with an heterogeneous coating of high emissivity black paint, the non-painted zones reflecting a low temperature surrounding called black body witch can be a real black body or any other device at a constant known cold temperature with high emissivity coating. Hence, schematically, the black spots emit IR radiations representative of the sample temperature, whereas the unpainted part reflects the black body low temperature. Consequently (as shown in figure 1a), sufficient gray contrast on IR images is obtained to follow the displacements and in the meantime an image of temperature is visible in black painted zones.

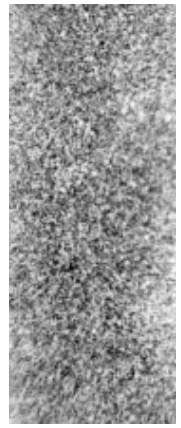

$-\mathrm{a}-$

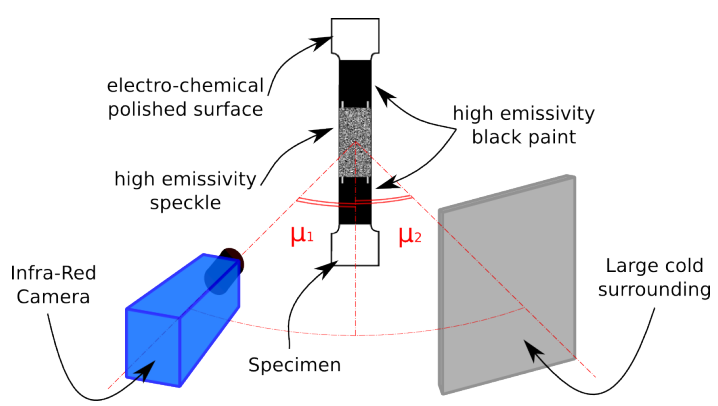

$-\mathrm{b}-$

Fig. 1. (a) Speckled sample face as observed at ambiant temperature with the IR camera in the set-up shown schematically on the right (b) 
From a practical point of view, the chosen experimental setup consists in an IR camera, tilted with a low angle from the specimen face normal, and a black body, also tilted with a low angle, reflecting in the specimen face (figure 1b).

With this set-up, we may follow the in-plane displacement of the sample thanks to sharp contrast, but due to emissivity variations on the surface, prior calibration of temperature measurement is required.

\section{Temperature calibration via cooling test}

To calibrate temperature measurement on our emissivity contrasted sample, we perform a cooling test on this sample with his own speckle-pattern and in the same environment and position as for the real test : testing machine, grips, IR camera and black-body (at the same low temperature).

In order to provide a validation of the calibration procedure, the back face of the sample, covered with a black uniform high emissivity coating, is reflected in a specific IR mirror in order to see both sample faces on the same camera image. Considering that the temperature is uniform across the thickness of the sample, the back face allows one to evaluate the temperature field that can be compared with the temperature calculated from the front face. The reflection of the mirror has been preliminary qualified by using the same imaging set up on a sample whose both faces were covered with a uniform high emissivity coating. Physical reference marks are drawn on each face to permit registration of both temperature fields.

As in any other IR measurement, the translation of the camera gray levels (Digital Levels or DL) into Celsius degree $\left({ }^{\circ} \mathrm{C}\right)$ temperature for a unit emissivity necessitates a prior calibration over the relevant range of temperature. Rather than using the built-in correspondence provided with the camera, a standard calibration has been performed using a reference black body over the temperature interval $5-60^{\circ} \mathrm{C}$.

This correspondence can be used directly on a surface with an emissivity close to unity, and the final calibration step is to evaluate variation of the DL with the local sample temperature for the image of the speckled specimen face in the specific set-up. The unloaded specimen cooling naturally from $60^{\circ} \mathrm{C}$ down to ambient temperature is imaged with the IR camera. The final (homogeneous) temperature is $T_{0} \approx 26{ }^{\circ} \mathrm{C}$. It is noticed that the observed image $f(\boldsymbol{x}, T(\boldsymbol{x}))$ (where $f$ is the DL value at pixel $\boldsymbol{x}$ ) at all times and temperatures obeys the following relation

$$
f(\boldsymbol{x}, T(\boldsymbol{x}))=\theta+\left((T-\theta) /\left(T_{0}-\theta\right)\right) f\left(\boldsymbol{x}, T_{0}\right) .
$$

The temperature $\theta$ can be understood as that of the black body. Indeed, if the sample was brought to the same temperature as the reflecting black body, the surface would appear as uniform, irrespective of its local emissivity. Thus, a single reference image of the surface at the reference temperature $T_{0}$ is sufficient to appreciate quantitatively the rendering of the surface for any temperature. In the above equation, all temperatures are expressed in DL, and the calibrated correspondence is used to express it in Celsius degrees. From a best fit of the above relation over the entire cooling period provides an independent measurement of $\theta=5.37^{\circ} \mathrm{C}$, consistent with the imposed value.

\section{Infra-Red image correlation equations}

Now that the image texture evolution with temperature is known, it can be exploited in order to generalize the standard brightness conservation principle which is at the basis of DIC. The issue is now to retrieve both the displacement field $\boldsymbol{u}(\boldsymbol{x})$ and temperature field $T(\boldsymbol{x})$ from the registration of an observed IR image, $g(x)$ under thermo-mechanical loading and a reference one (which we choose to be $f\left(\boldsymbol{x}, T_{0}\right)$ ), exploiting the following relation

$$
g(\boldsymbol{x})=f(\boldsymbol{x}+\boldsymbol{u}(\boldsymbol{x}), T(\boldsymbol{x}+\boldsymbol{u}(\boldsymbol{x}))) .
$$


So that, both unknown fields $T$ and $\boldsymbol{u}$ are obtained from the minimization of the following functional

$$
\mathcal{R}=\int\left[g(\boldsymbol{x})-\theta-\left((T(\boldsymbol{x}+\boldsymbol{u}(\boldsymbol{x}))-\theta) /\left(T_{0}-\theta\right)\right) f\left(\boldsymbol{x}+\boldsymbol{u}(\boldsymbol{x}), T_{0}\right)\right]^{2} \mathrm{~d} \boldsymbol{x}
$$

We have implemented the global DIC approach introduced in Ref. [6]. Both temperature and displacement field are decomposed over Q4 finite element shape functions (bilinear variation over square shaped elements). The algorithm proceeds through successive linearizations of the bracketed expression in the following functional around the current determination of the $(T, \mathbf{u})$ fields down to convergence. A multi-scale algorithm is implemented to allow for large scale displacements without secondary minima trapping, and achieve a quick and robust solution [7].

\section{Application : strain and temperature localization in SMA}

\subsection{Specific behavior of $\mathrm{NiTi}$}

NiTi based SMA are perfect candidates to evaluate this new method. Their specific behavior is due to a martensitic phase transformation - austenite at high temperature / martensite at low temperature - which can be activated either by mechanical loading or temperature variations. The superelastic behavior for instance is observed during mechanical loading in the austenite phase : after an "elastic" behavior, the stress-strain curve presents a plateau corresponding to austenite $\rightarrow$ martensite transformation.

These phenomena are well-known [8], [4] to be accompanied by strain localization, essentially on NiTi samples of various shapes (flat bone-shaped, thin tubes, wires) loaded in tension. The localization patterns generally form bands for flat samples [9] and helices for thin tubes [10], [11], [12], [13], [14], inclined at a 55 degree angle with the tension direction. The nucleation of multiple or a single band(s) and their propagation are sensitive to the imposed global strain rate [5]. The von Mises equivalent strain into bands was observed to be of the order of $3.5 \%$ whereas the equivalent strain outside the bands remains lower than $1.5 \%$ [9]. At the same time, the sample global temperature elevation may reach typical values of $20^{\circ} \mathrm{C}$, due to transformation latent heat, while heterogenety magnitude is about $8^{\circ} \mathrm{C}$ due to localization. These values obviously depend although on the specimen geometry and environment [15].

\subsection{Experimental procedure}

The experimental method presented above is applied to tensile tests on a NiTi SMA. The material of composition Ti-Ni 55.4 wt\% (commercial name SE508) was produced by Nitinol Devices and Components (Fremont, California, USA). Samples were formed by Nitifrance (Lury-sur-Arnon, France). The forming consists in a cold-rolling followed by a heat treatment of 2 minutes at $480^{\circ} \mathrm{C}$ in salt bath. Sample have a rectangular section of $20 \times 2 \mathrm{~mm}^{2}$ and a gauge zone length of $120 \mathrm{~mm}$.

The transformation temperature of the alloy were estimated using Differential Scanning Calorimetry (DSC) measurement : austenite start $A_{s}=3^{\circ} \mathrm{C}$, austenite finish $A_{f}=30^{\circ} \mathrm{C}$, martensite start $M_{s}=30^{\circ} \mathrm{C}$ and martensite finish $M_{f}=0^{\circ} \mathrm{C}$. No trace of rhombohedral phase (R-phase) is observed for this specific forming and heat treatment.

Test is performed on MTS hydraulic testing machine (capacity $100 \mathrm{kN}$ ). Loading force and global displacement are measured. Loading is displacement controlled, with rate set to $2 \mathrm{~mm} / \mathrm{min}$ and a maximum displacement value of $10 \mathrm{~mm}$. The test is performed at ambient temperature $\left(25^{\circ} \mathrm{C}\right)$, the material being consequently initially in the austenitic state. These values were chosen to be consistent with previous test results, performed on similar samples [16].

Because of the machine and the type of grip that are used, the sample has to be thermally isolated from the external heat sources, namely the surface of contact between the specimen and the hydraulics grips and the hydraulic actuator has to be considered. We interpose insulating plastic sheets between 
the specimen and the grips. Because of the significant applied tension forces, these insulants may give rise to a small amount of slip between the jaws and the specimen head.

An IR camera, Jade $I I I$ produced by $\mathrm{CEDIP}^{\circledR}$, allows us to obtain images with an acquisition frequency of $100 \mathrm{~Hz}$ and an integration time of $930 \mu \mathrm{s}$. In the present experiment, the physical size of a pixel is about $210 \mu \mathrm{m}$.

\subsection{Results and interpretation}

The obtained global stress - global strain curve is given in figure 2. Global strain means here actuator displacement relative to the initial length of the sample. The observed plateau is specific to superelastic behavior, i.e. stress induces austenite to martensite transformation. Such materials, prone to localization usually display a plateau behavior which should not be confused with the actual local constitutive law.

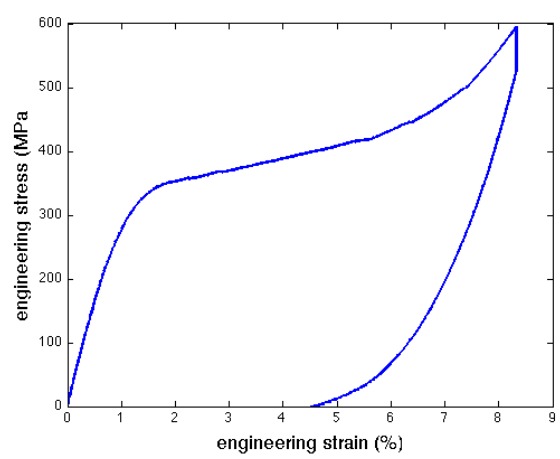

Fig. 2. Stress-strain curve for the analyzed test.

In the sequel we report on the analysis of a single IR image taken (no time averaging) in the plateau regime at the onset of the first visible shear band, at about $2.5 \%$ engineering strain. The size of the element was chosen to be 10 pixel (about $2 \mathrm{~mm}$ ) wide. Larger elements smear out the shear band over a larger region, whereas smaller ones display a significant amount of fluctuations.

Figure 3 shows (a) the region of interest of the reference image (prior to loading, after cooling period) as imaged with the IR camera and (b) a similar region of the specimen under load at the onset of the first localization band. In (c), image (b) corrected by the measured displacement field and effect of temperature elevation is to be compared with (a). The difference between this corrected image and the reference one is shown in (d) with the same gray level dynamics as the reference image. The uniformity of this difference signals a successful registration of the images. It is to be emphasized that the obvious temperature rise along the transformation band in (b) is mostly erased after the correction (c).

The temperature field obtained from the generalized DIC procedure outlined above are shown in Fig. 4(a). The temperature field has been converted to Celsius degrees. The estimated temperature rise within the shear band is about $5^{\circ} \mathrm{C}$ above that of the bulk, which is itself roughly $5^{\circ} \mathrm{C}$ higher than the initial temperature at this stage of loading (a higher final temperature is reached at the end of loading). The displacement field is shown in (b) and (c) after removal of the mean rigid body motion. The shear band appears markedly on both components of the displacement. Strains are more sensitive to noise, especially for small element sizes (since the number of pixels contributing to the determination of the displacement decreases). Nevertheless both the transverse and axial strains shown in (d) and (e) clearly reveal the presence of a shear band. The order of magnitude of the measured strain both inside and outside the band is in good agreement with previously published values on a similar NiTi material. 

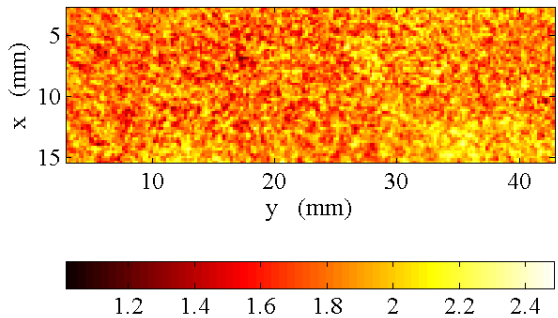

$-\mathrm{a}-$
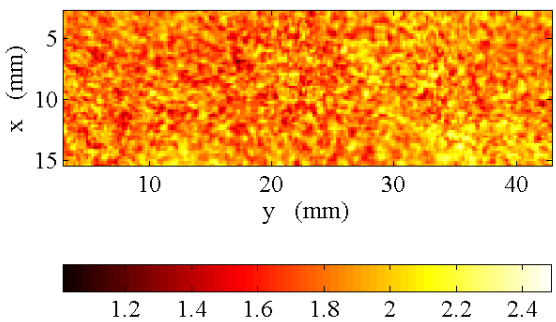

$-\mathrm{c}-$
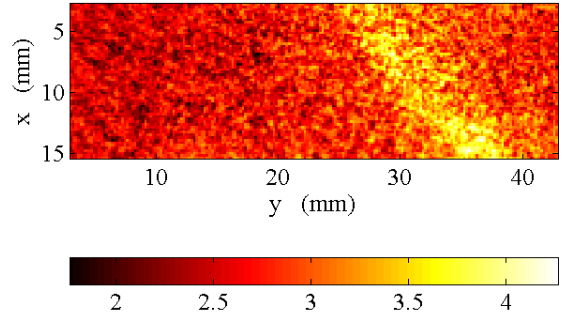

$-\mathrm{b}-$
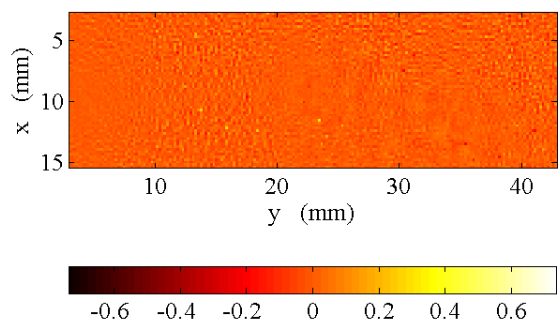

$-\mathrm{d}-$

Fig. 3. (a) Reference and (b) deformed images. The corrected deformed image and its difference with the reference, (or the residual), are shown respectively in (c) and (d).

\section{Conclusion}

A joint measurement of the kinematic and temperature fields stemming from an extended Digital Image Correlation technique has been presented. Although the present study reported on a single image, the entire time development of the sample deformation and the intermittency of shear banding is accessible. Similar spatial resolution to conventional global DIC can be reached, albeit an additional (temperature) field is estimated. One of the advantages of the present procedure, in addition to the greatly simplified experimental test set-up, is the fact that the relevant thermo-mechanical fields are directly evaluated onto a finite element mesh, and hence the interface with numerical simulations aiming at identification does not involve any unwanted loss of accuracy in unnecessary projection steps for the comparison. 


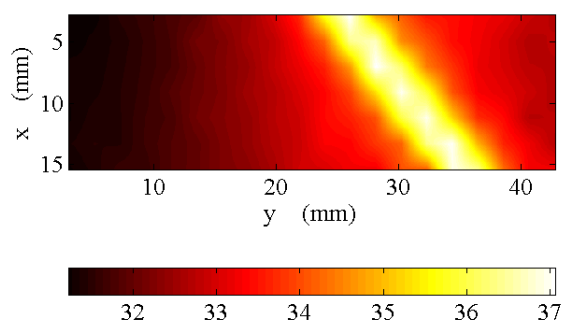

$-\mathrm{a}-$
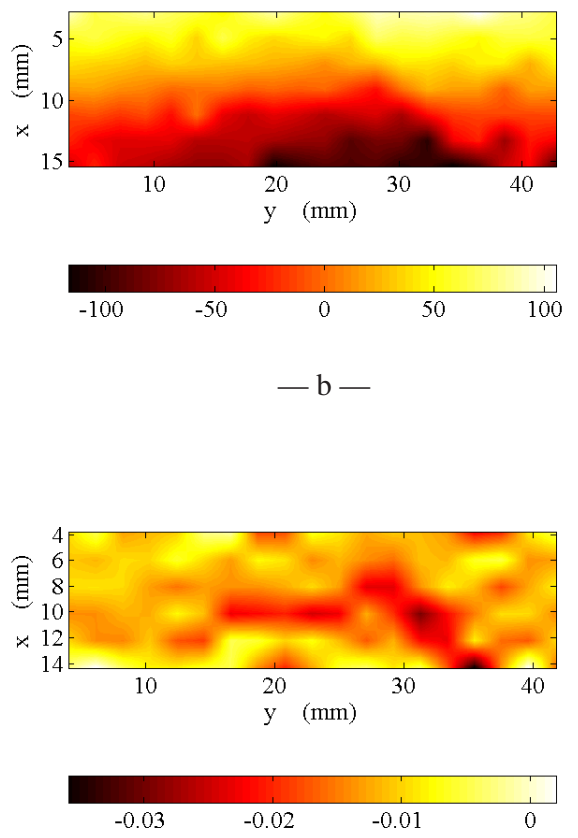

$-\mathrm{d}-$
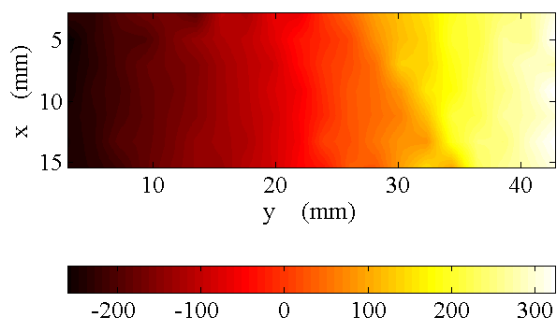

$-\mathrm{c}-$
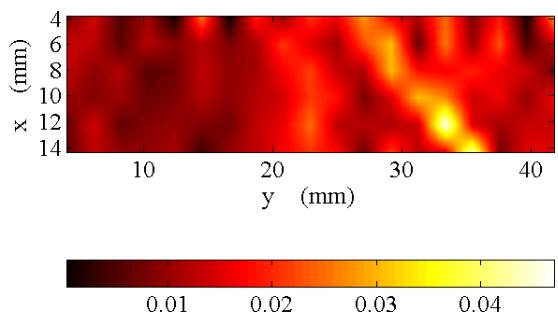

$-\mathrm{e}-$

Fig. 4. (a) Temperature field in Celsius degrees. (b) Transverse and (c) longitudinal displacements in micrometers. (d) and (e) show respectively the transverse $\left(\varepsilon_{x x}\right)$ and longitudinal $\left(\varepsilon_{y y}\right)$ strain fields.

\section{References}

1. A. Chrysochoos, B. Berthel, F. Latourte, G. Galtier, S. Pagano, B. Wattrisse, Journal of Strain Analysis for Engineering Design, 43, (2008), 411-421

2. L. Bodelot, L. Sabatier, E. Charkaluk, P. Dufrénoy, Material Science and Engineering, A 501, (2009), 52-60

3. J.J. Orteu, Y. Rotrou, T. Sentenac, L. Robert, Experimental Mechanics 48,(2008), 163-179

4. P. Sittner, Y. Liu, V. Novak, Journal of the Mechanics and Physics of Solids 53, (2005), 1719-1746

5. E.A. Pieczyska, S.P. Gadaj, W.K. Nowacki, H. Tobushi, Experimental Mechanics 46, (2006), 531542 
6. G. Besnard, F. Hild, S. Roux, Experimental Mechanics 46, (2006), 789-803

7. F. Hild, B. Raka, M. Baudequin, S. Roux, F. Cantelaube, Appl. Optics 41, (2002), 6815-6828

8. Q.P. Sun, Z. Zhong, International Journal of Plasticity 16, (2000), 1169-1187

9. S. Daly, G. Ravichandran, K. Bhattacharya, Acta Materialia 55, (2007), 3593-3600

10. Q.P. Sun, Z.Q. Li, International Journal of Solids and Structures 39, (2002), 3797-3809

11. P. Feng, Q.P. Sun, Journal of the Mechanics and Physics of Solids 54, (2006), 1568-1603

12. D. Favier, H. Louche, P. Schlosser, L. Orgéas, P. Vacher, L. Debove, Acta Materialia 55, (2007), 5310-5322

13. P. Schlosser, H. Louche, D. Favier, L. Orgéas, Strain 43, (2007), 260-271

14. P. Schlosser, D. Favier, H. Louche, L. Orgéas, Advances in Science and Technology, 59, (2008), 140-149

15. S.P. Gadaj, W.K. Nowacki, E.A. Pieczyska, Infrared Physics and Technology 43, (2002), 151-155

16. K. Lavernhe-Taillard, A. Maynadier, M. Poncelet, A. Benallal, Proceeding of 19ème Congrès Français de Mécanique, Marseille, France, (2009) 